\title{
Etablierung eines Brustzentrums an einer interkulturellen Schnittstelle: Das Brustgesundheits- zentrum Brixen-Meran, Partner der Universitätsklinik Innsbruck
}

\author{
Florian D. Vogl $\left.\right|^{a}$ Sonia Prader ${ }^{b} \quad$ Arthur Scherer $^{b} \quad$ Herbert Heidegger $^{a}$

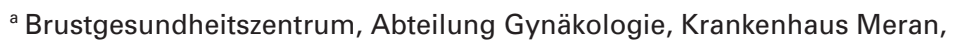 \\ ${ }^{\mathrm{b}}$ Brustgesundheitszentrum, Abteilung Gynäkologie, Krankenhaus Brixen, Italien
}

\section{Struktur und Gesundheitssystem in Südtirol}

Im Jahre 2006 wurde in Südtirol das Brustgesundheitszentrum Brixen-Meran (BGZ) erfolgreich nach den Vorgaben der Deutschen Krebsgesellschaft und Deutschen Gesellschaft für Senologie zertifiziert. Die Region Südtirol, amtlich Autonome Provinz Bozen, wurde 1919 mit dem Friedensvertrag von Saint Germain an Italien angeschlossen und nimmt heutzutage aufgrund ihres Autonomiestatus eine politische Sonderstellung in Italien ein. Die Bevölkerung beläuft sich aktuell auf knapp 500000 Einwohner, die zu zwei Dritteln deutschsprachig sind. Das Land verfügt über eine eigene Hochschule, die Freie Universität Bozen. Die nächsten medizinischen Fakultäten sind an der Landesuniversität Innsbruck und in Verona. Als Grenzland liegt Südtirol in einem kreativen Spannungsfeld zwischen Nord und Süd.

Zum Zeitpunkt der Vorbereitungen zur Zertifizierung des BGZ befand sich das Gesundheitssystem in Südtirol im Umbruch. Bis dahin gab es 7 öffentliche Krankenhäuser, mit unterschiedlichen Trägern, eigenen EDV Systemen und der damit verbundenen Schnittstellenproblematik. Da es sich um ein vorwiegend öffentliches Gesundheitssystem mit wenig Privatanteil handelt, stellte sich das Problem der langfristigen Finanzierbarkeit. Von Seiten der Landespolitik war das erklärte Ziel für die folgenden Jahre die Schaffung eines einheitlichen Sanitätsbetriebes für Südtirol und die Bildung von Schwerpunktzentren. Dabei wurde eine Anbindung an die Universität gewünscht. Durch diese Maßnahmen erhoffte man sich eine Qualitätssteigerung bei gleichzeitiger Kostendämmung.

Die Betreuung von Brustpatientinnen (ca. 300 Neuerkrankungen im Jahr) war bis dato nicht strukturiert. Der Erstkontakt durch eine Patientin erfolgte entweder über den Hausarzt, den Gynäkologen, oder andere Fachärzte, sowohl aus dem öffentlichen wie dem privaten Bereich; die Diagnosesicherung durch Radiologen, Chirurgen oder Gynäkologen.
Die stationäre Betreuung umfasste Staging und Operation durch Gynäkologen oder Chirurgen, und die Festlegung der weiteren Behandlung durch den jeweils betreuenden Facharzt. Patientinnen konnten sich in jedem der 7 öffentlichen und einem privaten Krankenhaus behandeln lassen.

Unsere Vision der Versorgung der Brustpatientin hingegen sah das Brustzentrum als Kompetenzzentrum, das eine Vernetzung der vorhandenen Strukturen, Verbesserung der interdisziplinären Kommunikation sowie Vermeidung von Doppelgleisigkeit ermöglicht, alles mit dem Ziel der Effizienzsteigerung einer leitlinienorientierten Versorgung zum Wohl der Patientinnen.

\section{Das Brustgesundheitszentrum Brixen-Meran}

Die Initiative zur Bildung des BGZ kam aus den Gynäkologischen Abteilungen des Krankenhauses Meran und des Krankenhauses Brixen, die nun die beiden wesentlichen Standorte des BGZ darstellen und die stationäre Versorgung der Brustpatienten gewährleisten. In beiden Krankenhäusern ist eine eigene Abteilung für diagnostische Radiologie vorhanden, in Meran auch eine interdisziplinäre onkologische Tagesklinik. Alle Patientinnen haben Zugang zu Schmerzdienst, Psychoonkologie, Sozialdienst, Seelsorge und genetischer Beratung. Auch können plastisch-chirurgische Eingriffe an beiden Standorten durchgeführt werden. Zentrale Abteilungen für Pathologie, Nuklearmedizin und Strahlentherapie sind seit jeher am Zentralkrankenhaus in der Landeshauptstadt Bozen angesiedelt und verantwortlich für die Versorgung der Bevölkerung der gesamten Provinz. Externe Kooperationspartner sind die Südtiroler Krebshilfe, die Selbsthilfegruppe mamazone.it (eine Tochter der deutschen mamazone e.V., die übrigens von einer Südtirolerin gegründet wurde), sowie das Sanitätshaus. Alle Partner sind durch Kooperationsverträge in das BGZ eingebunden.

\section{KARGER}

Fax +497614520714

Information@Karger.de

www.karger.com (c) 2009 S. Karger GmbH, Freiburg

Accessible online at:

www.karger.com/brc
Dr. Florian D. Vogl, MSc

Abt. Gynaekologie und Geburtshilfe

Krankenhaus «F. Tappeiner»

Rossinistrasse 12, 39012 Meran, Italy

Tel. +390473 26-7939, Fax -4190

florian.vogl@asbmeran-o.it 
Das Brustzentrum Tirol (Universität Innsbruck), das seinerseits 2003 zertifiziert wurde, tritt strukturell als wichtiger Partner für das BGZ Brixen-Meran auf (Abb. 1). Bereits in den Jahren vor der Zertifizierung bestand eine enge Zusammenarbeit, die nun über einen Kooperationsvertrag formalisiert wurde. Dies garantiert die Anbindung an die Landesuniversität Innsbruck und somit auch den Zugang zu internationalen Studien [1]. Darüber hinaus ist das BGZ an nationalen, d.h. italienischen, Studien beteiligt, und hat eigene wissenschaftliche Projekte etabliert. Die vielfältige Studienaktivität gewährleistet auch ein international anerkanntes Niveau der Therapie.

Die besonderen geographischen und kulturellen Gegebenheiten Südtirols brachten bei der Etablierung des BGZs einige spezifische Probleme mit sich, für die Lösungen gefunden werden mussten: Die 3 Städte Brixen, Bozen und Meran bilden auf der Landkarte die Eckpunkte eines etwa gleichschenkeligen Dreiecks, wobei die Distanz von Brixen und Meran nach Bozen jeweils kleiner als $45 \mathrm{~km}$ ist, zwischen den Standorten Brixen und Meran sich jedoch ein Berg befindet. Um dieses Hindernis zu überwinden wurde für die wöchentliche Tumorkonferenz ein Videokonferenzsystem eingerichtet, das es allen Behandlungspartnern in den beteiligten Kliniken, nämlich Brixen, Meran, Bozen, und die Partner der Universitätskliniken Innsbruck ermöglicht, gemeinsam und in «real time» Fälle besprechen können. Außerdem steht ein Tele-Pathologie-System zur Ad-hoc-Beurteilung von Schnellschnittpräparaten zur Verfügung. Für die beiden Standorte Brixen und Meran wurde ein Dokumentenserver zur Ablage gemeinsamer Formulare, SOPs, und Protokolle eingerichtet, wodurch die Dokumentenlenkung wesentlich erleichtert wird. Weiterhin verfügt das BGZ über ein gemeinsames TumorDokumentationssystem, so dass eine einheitliche Datenstruktur und gemeinsame Auswertung gewährleistet werden.

Ein kulturelles Problem ergab sich bei der Definition der Anlaufstelle für Brustpatientinnen. Während die «italienische Schule» den Chirurgen als Ansprechpartner sieht, ist nach der «deutschen Schule» der Gynäkologe der Senologe. Durch entsprechende Öffentlichkeitsarbeit wurde das «deutsche» Konzept beworben und von der Bevölkerung und den Kollegen offensichtlich akzeptiert.

\section{Stärken und Schwächen des BGZ}

Der Zusammenschluss aller öffentlichen Krankenhäuser Anfang des Jahres 2007 unter einen gemeinsamen Träger, den Südtiroler Sanitätsbetrieb, und das politische Streben nach Kompetenzzentren haben uns den Weg der Zertifizierung erleichtert. Knapp 3 Jahre nach der Erstzertifizierung lassen sich mehr als die Hälfte der Brustkrebspatientinnen in Südtirol im BGZ behandeln - mit zunehmender Tendenz. Die Steigerung der Fallzahl ist und bleibt eine Priorität unseres Handelns. Allerdings ist die Motivation hierfür die Qualitätssteigerung und nicht ein Konkurrenzdruck, wie er in anderen Ländern herrschen mag. Zahlreiche Studien belegen einen Überlebensvorteil für Patienten, die in qualitätsorientiert arbeitenden Zentren behandelt werden. Die strategisch günstige Lage von Meran und Brixen im westlichen bzw. östli-

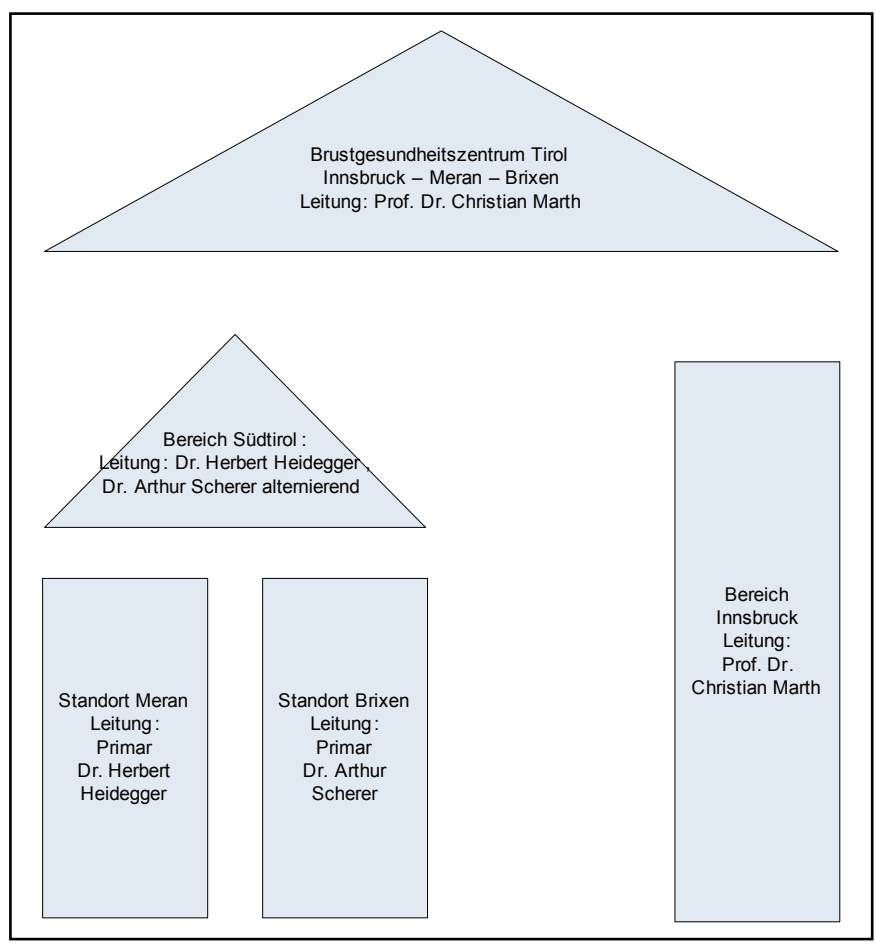

Abb. 1. Struktur des Brustgesundheitszentrums Brixen-Meran, Partner der Universität Innsbruck.

chen Teil des Landes erlaubt allen Südtiroler Frauen einen günstigen Zugang zum BGZ. Unter diesen Voraussetzungen wird sich die Brustkrebsbehandlung in den peripheren Krankenhäusern langfristig nicht halten lassen.

$\mathrm{Zu}$ den Stärken der Südtiroler Realität zählt sicherlich, dass alle Brustpatientinnen ausschließlich im Krankenhaus betreut werden. Dies gilt für die Diagnostik und operative Behandlung, genauso wie für die ambulante chemotherapeutische Behandlung und insbesondere für die Nachsorge, die - anders als in den meisten deutschsprachigen Ländern - ausschließlich im Krankenhaus durchgeführt wird. Dies erleichtert die Datensammlung für die Analysen zur Ergebnisqualität. Ein weiterer Vorteil ist der zentrale Dienst für Pathologie in der Landeshauptstadt Bozen, der eine einheitliche Diktion im histologischen Befund garantiert.

Für die Zukunft streben wir eine Ausdehnung und Bündelung der Kompetenzen unseres Zentrums an. Nach den Regeln der wirtschaftlichen Unternehmensführung dürfte damit eine erhebliche Kosteneinsparung verbunden sein.

Im Sinne unseres Leitbildes der qualitätsgestützten, kompetenzgetragenen Versorgung wollen wir die interdisziplinäre $\mathrm{Zu}$ sammenarbeit auf hohem qualitativem und technischem Niveau weiter optimieren. Erfreulich ist, dass auch in Südtirol die Senologie weitestgehend in der Hand des Gynäkologen bleibt und unseren Patientinnen die Betreuung von der Diagnose bis zur Nachsorge garantiert.

\section{Literatur}

1 Marth C, Frede T, Müller-Holzner E, et. al.: Brustgesundheitszentrum Tirol, Universitätskliniken, Innsbruck. Breast Care 2006;1:336-337. 